\title{
Recent-onset atrial fibrillation: a study exploring the elements of Virchow's triad after cardioversion
}

\author{
Panagiotis Arvanitis ${ }^{1}$ (D) Anna-Karin Johansson ${ }^{2} \cdot$ Mats Frick $^{2} \cdot$ Helena Malmborg $^{1} \cdot$ Spyridon Gerovasileiou $^{3}$. \\ Elna-Marie Larsson ${ }^{4} \cdot$ Carina Blomström-Lundqvist $^{1}$
}

Received: 24 May 2021 / Accepted: 10 October 2021 / Published online: 24 October 2021

(c) The Author(s) 2021

\begin{abstract}
Purpose Atrial fibrillation (AF) imposes an inherent risk for stroke and silent cerebral emboli, partly related to left atrial (LA) remodeling and activation of inflammatory and coagulation systems. The aim was to explore the effects of cardioversion (CV) and short-lasting AF on left atrial hemodynamics, inflammatory, coagulative and cardiac biomarkers, and the association between LA functional recovery and the presence of a prior history of AF.

Methods Patients referred for CV within $48 \mathrm{~h}$ after AF onset were prospectively included. Echocardiography and blood sampling were performed immediately prior, $1-3 \mathrm{~h}$ after, and at 7-10 days after $\mathrm{CV}$. The presence of chronic white matter hyperintensities $(\mathrm{WMH})$ on magnetic resonance imaging was related to biomarker levels.

Results Forty-three patients ( $84 \%$ males), aged 55 \pm 9.6 years, with median $\mathrm{CHA}_{2} \mathrm{DS}_{2}$-VASc score 1 (IQR 0-1) were included. The LA emptying fraction (LAEF), LA peak longitudinal strain during reservoir, conduit, and contractile phases improved significantly after CV. Only LAEF normalized within 10 days. Interleukin-6, high-sensitivity cardiac-troponin-T (hs-cTNT), N-terminal-pro-brain-natriuretic peptide, prothrombin-fragment $1+2(\mathrm{PTf} 1+2)$, and fibrinogen decreased significantly after $\mathrm{CV}$. There was a trend towards higher C-reactive protein, hs-cTNT, and PTf1+2 levels in patients with WMH $(n=21)$ compared to those without $(n=22)$. At 7-10 days, the LAEF was significantly lower in patients with a prior history of AF versus those without.

Conclusion Although LA stunning resolved within 10 days, LAEF remained significantly lower in patients with a prior history of AF versus those without. Inflammatory and coagulative biomarkers were higher before CV, but subsided after 7-10 days, which altogether might suggest an enhanced thrombogenicity, even in these low-risk patients.
\end{abstract}

Keywords Recent-onset atrial fibrillation · Left atrial remodeling · Inflammation and coagulation systems · White matter hyperintensities

Panagiotis Arvanitis

arvanitis.panagiotis@medsci.uu.se;

panagiotis.arvanitis@gmail.com

1 Department of Medical Science and Cardiology, Uppsala University, Sjukhusvägen 9, Ing 35, 75309 Uppsala, Sweden

2 Department of Clinical Science and Education, Division of Cardiology, Karolinska Institutet, South Hospital, Stockholm, Sweden

3 Department of Medical Sciences, Uppsala University, Clinical Physiology and Cardiology, Uppsala University, Uppsala, Sweden

4 Department of Surgical Science, Radiology, Uppsala University, Uppsala, Sweden

\section{Introduction}

The mechanism of thrombogenesis is complex and multifactorial and as described by Rudolf Virchow over a century ago, there are three main elements: (i) hemodynamic changes with abnormal blood flow, (ii) hypercoagulability, and (iii) endothelial dysfunction [1,2]. Atrial fibrillation (AF) carries an inherent risk for intracavitary thrombus formation and with time electrical, structural, and functional remodeling of the left atrium (LA) result in atrial mechanical stunning [3-5]. Cardiomyocyte apoptosis, endothelial injury, and replacement fibrosis attribute to irreversible remodeling, leading to LA dilatation and dysfunction [6]. The functional recovery, following restoration of sinus rhythm, depends on the extent of irreversible structural remodeling and is 
thought to contribute to the development of silent or clinically evident cerebrovascular thromboembolic events, as the majority occur within 10 days after restoration of sinus rhythm $[4,5,7-10]$. Virchow's triad of thrombogenicity is completed by the activation of inflammatory and coagulative system during AF. Although the activation of these systems may potentially explain the high incidence of silent, chronic white matter hyperintensities (WMH) observed on magnetic resonance imaging (MRI) in AF patients with $\mathrm{AF}$, it remains unclear whether microemboli, direct microvascular injury, or transient cerebral hypoperfusion play a role in the pathophysiologic mechanisms of WMH [11-14].

The aim of the study was to assess the effects of a shortlasting AF episode $(<48 \mathrm{~h})$ and electrical cardioversion (CV) on thrombogenicity in anticoagulant-naïve patients by analyzing LA hemodynamics and biomarkers reflecting hypercoagulability on various levels, furthermore to explore whether a prior history of AF affects the degree of atrial functional recovery.

\section{Methods}

\subsection{Study population}

Patients with recent onset of AF within $48 \mathrm{~h}$, naïve to oral anticoagulant and eligible for rhythm control treatment with $\mathrm{CV}$, were prospectively included in the study; the details of which have been described previously [15]. In brief, patients were recruited from the emergency departments of two university hospitals in Sweden. Exclusion criteria were known moderate to severe valvular heart disease, previous or acute cerebrovascular event or thromboembolism, moderate or severe left ventricular systolic dysfunction, known coagulation defects, and contraindications for MRI and CV for $\mathrm{AF}$ in the preceding 3 months. Electrical $\mathrm{CV}$ was performed without heparin or low molecular weight heparin periprocedurally. The study was approved by the Regional Ethical Review Board and complied with the Declaration of Helsinki. Written informed consent was obtained from each study participant.

\subsection{Endpoints}

The primary objective was to assess the effects of a shortlasting AF episode and CV on LA size, function, and myocardial deformation by echocardiography as well as on the inflammatory, coagulative, and cardiac/endothelial systems, by measuring corresponding biomarkers in anticoagulantnaïve patients with recent-onset $\mathrm{AF}$ and further to explore the association between the degree of atrial functional recovery and the presence of prior history of AF. The finding of WMH on brain MRI, as described in our previous study, urged us to further explore its relation to coagulative and inflammatory biomarkers [15].

In order to cover the time intervals associated with LA reverse remodeling and functional recovery, the echocardiographic examinations and blood sampling were scheduled within $2 \mathrm{~h}$ prior to $\mathrm{CV}, 1-3$ hours after, and at 7-10 days after $\mathrm{CV}$, representing the recovery period of the LA contractile function with the highest thromboembolic events [4, 5, 8, 15]. The last follow-up was at 30 days after CV (Fig. 1).

\subsection{Echocardiographic assessment}

Transthoracic echocardiography was performed using General Electric Vivid E9 ultrasound system and according to recommendations by the European Association of Cardiovascular Imaging [16, 17]. Left atrial volumes were measured using the modified Simpson's method at the apical four-chamber view. The LA maximal end-diastolic volume (LAEDV) was measured at the beginning of cardiac diastole, the end-systolic volume (LAESV) at the end of cardiac diastole, and the total emptying fraction (LAEF) was calculated as (LAEDV-LAESV)/LAEDV $\times 100$. The LA volume indexed to body surface area (LAVI) was also calculated. Speckle tracking echocardiography (STE) for LA deformation analysis was assessed offline using General Electric EchoPAC software version 201. The LA peak longitudinal strain during reservoir phase at the end of ventricular diastole until mitral valve opening (LAcR), during conduit phase until the onset of atrial contraction (LAECD), and during contraction phase until the end of the ventricular

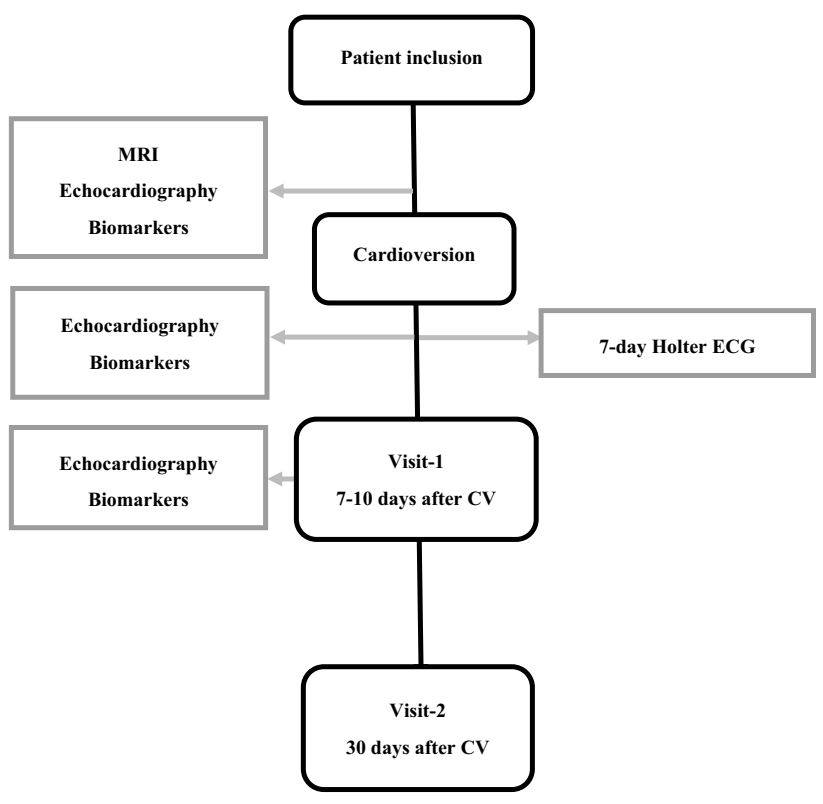

Fig. 1 Study flowchart. CV: cardioversion; ECG: electrocardiography; MRI: magnetic resonance imaging. 
diastole (LAcCT) were measured. The zero strain reference was set at left ventricular end-diastole. Left ventricular volumes were measured at the end of left ventricular diastole (LVEDV) and systole (LVESV) using the modified Simpson's method and left ventricular ejection fraction (LVEF) was calculated as (LVEDV-LVESV)/LVEDV $\times 100$. Echocardiographic variables were compared in patients with and without the presence of a history of paroxysmal or persistent AF. All measurements were made by one physician experienced in echocardiographic imaging and blinded to patient demography. The values were the mean of 3 consecutive measurements.

\subsection{Magnetic resonance imaging}

The brain MRI was performed prior to $\mathrm{CV}$ as described earlier [15]. In brief, chronic deep white matter lesions or white matter hyperintensities were visually quantified using the Fazekas scale where 0 equals normal, 1 is assigned to punctate lesions, and 3 is assigned to the most extreme cases where extensive and confluent lesions are present [18]. Patients with and without WMH were compared with regard to levels of inflammatory and coagulative biomarkers.

\subsection{Biomarkers}

Blood sampling for biomarkers was performed $2 \mathrm{~h}$ prior to $\mathrm{CV}, 1-3 \mathrm{~h}$, and 7-10 days after $\mathrm{CV}$. Inflammatory biomarkers included interleukin-6 (IL-6), C-reactive protein (CRP), and P-selectin. Cardiac biomarkers included highsensitivity cardiac troponin $\mathrm{T}$ (hs-cTNT) and N-terminal pro-brain natriuretic peptide (NT-proBNP). Coagulation biomarkers included prothrombin fragment 1+2 (PTf1+2), von Willebrand Factor antigen (vWFag), coagulation factor VIII, fibrinogen, and D-dimer. Specific methods for analyzing biomarkers are presented in supplement table 1.

\subsection{Statistical analysis}

The variables were tested for normality using Shapiro-Wilk test. The results were reported as mean \pm standard deviation (SD) for normally distributed variables, as median (interquartile range) for non-normally distributed variables and counts, $n$, and percentage (\%) for the remaining. Normally distributed variables were analyzed using Student $t$ test for pairwise comparison and repeated measures analysis of variance (rmANOVA) with the use of Greenhouse-Geisser or Huynh-Feldt correction for violation of Mauchly's test of sphericity for serial results analysis. Variables with a nonnormal distribution were analyzed using Wilcoxon signedrank test for their pairwise comparisons, adjusting significance values by the Bonferroni correction for multiple tests, and Friedman's two-way analysis of variance by ranks for serial result analysis. A value of $p<0.05$ was considered significant. Missing values classified as "missing at random" were treated with single imputation [19]. Left-censored data below the lower limit of quantification (LOQ) were treated with simple substitution with $\mathrm{LOQ} / \sqrt{2}$. Repeatability analysis was performed on 42 echocardiographic examinations by two raters (SG and PA), at two different time points, using the intraclass correlation coefficient (ICC). A twoway random-effect model based on average ratings and absolute agreement assessed the inter-rater repeatability. Mean estimations along with $95 \%$ confidence intervals (CI) were reported for each ICC; interpretation was as follows: $<0.50$, poor; between 0.50 and 0.75 , fair; between 0.75 and 0.90 , good; above 0.90 , excellent. All statistics were analyzed with IBM Statistical Package for the Social Sciences, version 27 for Windows (IBM Corp. Released 2017. IBM SPSS Statistics for Windows, Armonk, NY).

\section{Results}

\subsection{Study population}

Forty-three patients were included, of whom 26/43 (60.5\%) had a prior history of paroxysmal or persistent AF (Table 1). The $\mathrm{CHA}_{2} \mathrm{DS}_{2}$-VASc score [20] was $\leq 1$ in $40(93.8 \%)$ patients. Six patients $(6 / 43)$ had undergone $\mathrm{CV}$ within twelve months (4-8.8 months) prior to inclusion; two of which have had persistent AF.

Forty-one patients were successfully cardioverted while 2 patients converted spontaneously to sinus rhythm after the first MRI examination. The 7-day Holter monitoring after CV-detected AF recurrence in 4/43 (9.3\%) patients of whom 3 had paroxysmal AF with durations 33-183 s and one had persistent AF scheduled for repeat elective CV. Seven of the $43(16.2 \%)$ patients were initiated on novel oral anticoagulants after $\mathrm{CV}$ and continued medication during the study period; 3 of these patients had $\mathrm{CHA}_{2} \mathrm{DS}_{2}$-VASc score $\geq 2$ and 4 patients had score 1 . Another patient received oral anticoagulants on the 6th day due to peripheral emboli related to Leiden mutation.

Missing data, categorized as randomly missing, reached $14.5 \%$.

\subsection{Echocardiographic assessment}

The baseline LAVI was normal in all patients. There were no significant differences in the serial LAVI measurements prior $\mathrm{CV}, 1-3 \mathrm{~h}$ after and at 7-10 days after $\mathrm{CV}$ using rmANOVA. There was a statistically significant overall decrease in LAESV from prior $\mathrm{CV}, 1-3 \mathrm{~h}$ after $\mathrm{CV}$ to the 7-10 days after CV. Consequently, there was a corresponding statistically significant overall improvement in LAEF, 
Table 1 Basic characteristics of study population

\begin{tabular}{ll}
\hline Variables & Patients $n=43$ \\
\hline Gender, males & $36(83.7)$ \\
Age, yrs. & $55 \pm 9.6$ \\
BMI, kg/m ${ }^{2}$ & $26 \pm 3.4$ \\
Hypertension & $9(20.9)$ \\
Diabetes Mellitus & $2(4.7)$ \\
Vascular disease & $1(2.3)$ \\
Systolic BP (mmHg) & $125 \pm 13$ \\
Diastolic BP (mmHg) & $81 \pm 9$ \\
Plasma Creatinine, $\mu$ mol/L & $82.8 \pm 13.5$ \\
$\mathrm{CHA}_{2} \mathrm{DS}_{2}$-VASc $=0$ & $21(48.8)$ \\
$\mathrm{CHA}_{2} \mathrm{DS}_{2}$-VASc $=1$ & $19(44.2)$ \\
$\mathrm{CH}_{2} \mathrm{DS}_{2}$-VASc $\geq 2$ & $3(6.9)$ \\
First-onset AF & $17(39.5)$ \\
Previous history of paroxysmal AF & $17(39.5)$ \\
Previous history of persistent AF & $9(20)$ \\
AF duration from symptom onset to diagnosis, hours & $13.3 \pm 11.7$ \\
Heart rate at presentation (bpm) & $110 \pm 31$ \\
ACE/ARB medication & $8(18.6)$ \\
Antiplatelet therapy & $0(0)$ \\
Beta receptor blockers & $9(20.9)$ \\
\hline
\end{tabular}

Figures are mean \pm one standard deviation or frequencies and percentages; $A C E$, angiotensin-converting enzyme; $A F$, atrial fibrillation; $A R B$, aldosterone receptor blocker; $B M I$, body mass index; $B P$, blood pressure; $C V$, cardioversion; $K g$, kilogram; $m$, meter; $m l$, milliliter; yrs., years from prior $\mathrm{CV}, 1-3 \mathrm{~h}$ after $\mathrm{CV}$ to $7-10$ days after $\mathrm{CV}$ using rmANOVA, which remained stable for both patients with and without prior history of AF (Table 2). Although the LAEF normalized at 7-10 days, LAEF at 7-10 days was significantly lower in those with versus those without a prior AF history, 50.3 \pm 8.8 versus $56.3 \pm 8.7$, respectively, $p=0.032$. A statistically significant improvement in all three LA deformation indices, $\mathrm{LA} \varepsilon \mathrm{R}, \mathrm{LA} \varepsilon \mathrm{CD}$, and $\mathrm{LA} \varepsilon \mathrm{CT}$, for the entire cohort, and a trend of lower values at baseline were observed in those with a prior AF history. Moreover, when comparing LA function after restoration of sinus rhythm at $1-3 \mathrm{~h}$ versus $7-10$ days, there was a statistically significant increase of the LAEF $\mathrm{t}(42)=-2.4 p=0.023$, LA $\varepsilon \mathrm{R} t(42)=$ $-4.4 p<0.001$, LA $\varepsilon$ CD $t(42)=-2.7 p=0.009$, and LA $\varepsilon$ CT $t(42)=-4.2 p<0.001$.

Although in all study participants the LVEDV, LVESV, and LVEF were all within the normal ranges prior to $\mathrm{CV}$, the LVEF further improved significantly after CV (Fig. 2). Significantly higher LVEDV was also observed after CV, as compared to prior to CV. Moreover, the increase in LVEDV was independent of the ventricular rate during AF, even when the cohort was dichotomized by the ventricular rate below or above 110 beats per minute.

The ICC for inter-rater reliability was between fair and good; detailed results are presented in supplement table 2.
Table 2 Echocardiographic variables before and after electrical cardioversion

\begin{tabular}{|c|c|c|c|c|c|}
\hline $\begin{array}{l}\text { Echocar- } \\
\text { diographic } \\
\text { parameter }\end{array}$ & Prior to $\mathrm{CV}$ & After CV & 7-10 days after $\mathrm{CV}$ & Repeated measure ANOVA & $p$ value \\
\hline $\operatorname{LAVI}\left(\mathrm{mL} / \mathrm{m}^{2}\right)$ & $31.3 \pm 9.4$ & $32.3 \pm 9.3$ & $31.8 \pm 10.9$ & $F(1.89,76.4)=0.29$ & 0.722 \\
\hline LAEDV (ml) & $62.4 \pm 20.6$ & $67.0 \pm 19.9$ & $64.9 \pm 22.1$ & $F(2,84)=1.34$ & 0.267 \\
\hline LAESV (ml) & $38.9 \pm 13.9$ & $34.9 \pm 14.2$ & $31.1 \pm 13.3$ & $F(2,84)=10.47$ & $<0.001$ \\
\hline $\operatorname{LAEF}(\%)$ & $37.3 \pm 12.5$ & $48.7 \pm 9.7$ & $52.7 \pm 9.2$ & $F(1.83,76.8)=30.75$ & $<0.001$ \\
\hline $\mathrm{LA} \varepsilon \mathrm{R}(\%)$ & $11.9 \pm 7.0$ & $20.5 \pm 5.2$ & $25.6 \pm 6.8$ & $F(2,84)=56.99$ & $<0.001$ \\
\hline $\mathrm{LA \varepsilon CD}(\%)$ & $-7.7 \pm 5.0$ & $-11.4 \pm 3.2$ & $-13.2 \pm 4.0$ & $F(1.73,72.75)=23.63$ & $<0.001$ \\
\hline $\mathrm{LA} \varepsilon \mathrm{CT}(\%)$ & N/A* & $-9.0 \pm 3.3$ & $-12.1 \pm 4.5$ & $t(42)=-2.4^{\S}$ & 0.023 \\
\hline LVEDV (ml) & $87.4 \pm 21.2$ & $112.2 \pm 16.4$ & $117.5 \pm 21.2$ & $F(2,84)=55.97$ & $<0.001$ \\
\hline LVESV (ml) & $39.2 \pm 11.2$ & $49.3 \pm 12.6$ & $46.3 \pm 10.6$ & $F(2,84)=13.55$ & $<0.001$ \\
\hline $\operatorname{LVEF}(\%)$ & $55.9 \pm 8.4$ & $56.2 \pm 7.4$ & $60.5 \pm 6.4$ & $F(1.79,75.11)=7.76$ & 0.001 \\
\hline
\end{tabular}

Figures are mean \pm standard deviation.

*Non-applicable during atrial fibrillation

${ }^{\S}$ Paired sample $t$-test

$A N O V A$, analysis of variance; $C V$, cardioversion; $L A E D V$, left atrial end-diastolic volume; $L A E S V$, left atrial end-systolic volume; $L A E F$, left atrial total emptying fraction; $L A \varepsilon R$, left atrial peak longitudinal strain during reservoir function; $L A \varepsilon C D$, left atrial peak longitudinal strain during conduit function; $L A \varepsilon C T$, left atrial peak longitudinal strain during contractile function; $L A V I$, left atrial volume index; $L V E D V$, left ventricular end-diastolic volume; $L V E S V$, left ventricular end-systolic volume; $L V E F$, left ventricular ejection fraction; $m l$, milliliter; $m$, meter 
Fig. 2 Left atrial and left ventricular ejection fraction. Graphs represent ordinary mean plots and interaction plots, error bars, $95 \%$ confidence interval; groups according to positive or negative atrial fibrillation history; AF: atrial fibrillation; CV: cardioversion; LAEF: left atrial emptying fraction; LVEF: left ventricular ejection fraction; Par.AF: paroxysmal atrial fibrillation; Per.AF: persistent atrial fibrillation.

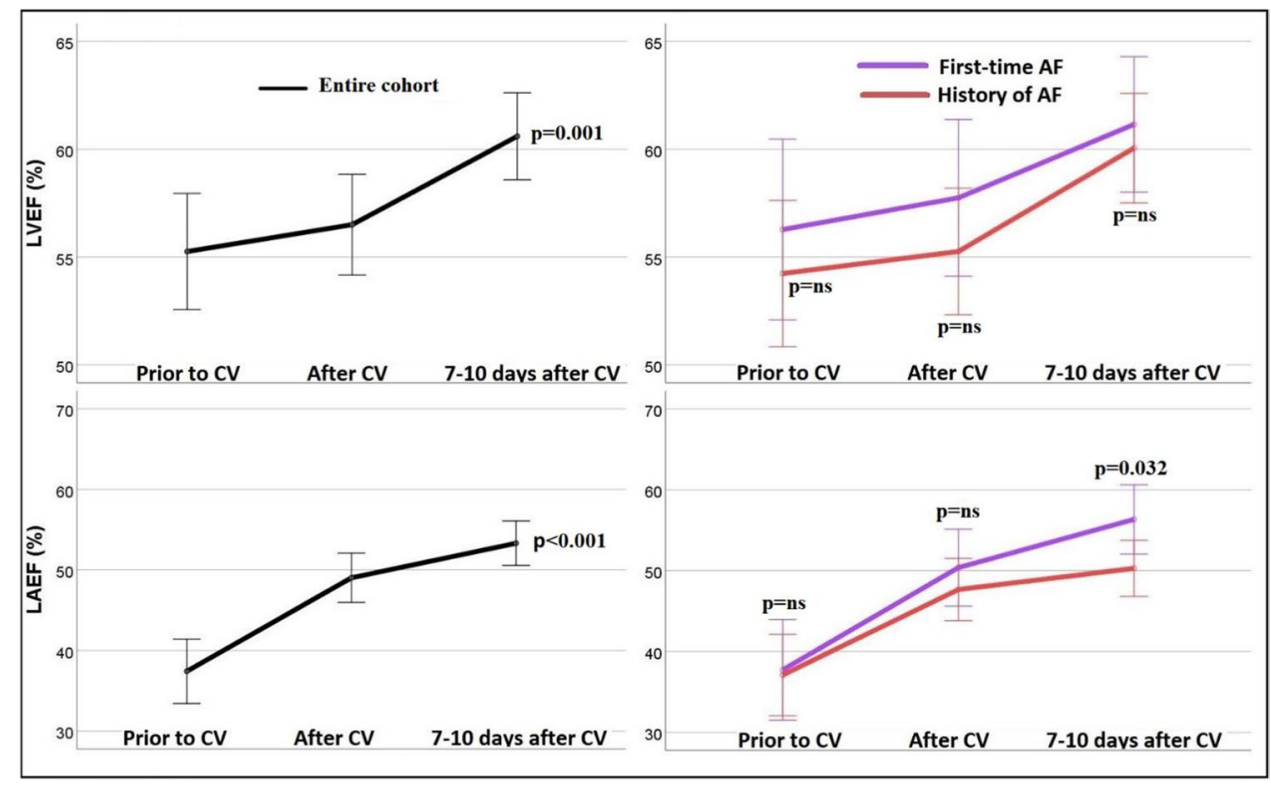

\subsection{Inflammatory, cardiac, and coagulative biomarkers}

Amongst biomarkers of inflammation and endothelial dysfunction, IL-6 and P-selectin showed a statistically significant overall decrease in the serial measurement analysis from prior to $\mathrm{CV}$ to the 7-10 days after CV. The CRP did not change significantly. Both cardiac biomarkers, hs-cTNT and NTpro-BNP, decreased statistically significantly, after CV. The biomarkers of coagulation, PTf $1+2$ and fibrinogen, decreased statistically significantly in the serial analysis from prior to $\mathrm{CV}$ to the 7-10 days after CV. The vWfAg increased transiently after $\mathrm{CV}$ and decreased 7-10 days after $\mathrm{CV}$, showing an overall statistically significant change in serial analysis. The FVIII and D-Dimer did not change significantly (Table 3 ). The levels of observed biomarkers did not differ in patients with versus without prior history of $\mathrm{AF}$, or in patients spontaneously versus electrically cardioverted.

\subsection{Chronic white matter hyperintensities}

Brain MRI prior to CV showed chronic white mater lesions or white matter T2 hyperintense lesions in 21/43 (49\%) of the patients, of whom 18/43 (42\%) had Fazekas score 1 and 3 (7\%) Fazekas score 2. Patients with WMH showed a tendency of higher CRP, hs-cTNT, NTpro-BNP, and PTf1+2 as compared with patients without WMH, but the difference did not reach statistical significance (Fig. 3). No association was found between the presence of previous AF history and presence of WMH. No patient had signs of recent acute ischemic lesions.

\section{Discussion}

In this prospective observational study with serial echocardiographic examinations for the evaluation LA stunning in conjunction with blood sampling of various biomarkers reflecting inflammatory, coagulative, and cardiac changes peri- and post-cardioversion, we were able to detect signs of enhanced thrombogenicity, even in low-risk patients with recent onset AF. The inclusion of only anticoagulant-naïve patients eliminated interactions related to the coagulative system making interpretations easier.

Abnormal blood flow, the first element of Virchow's triad, corresponds to the left atrial hemodynamics. Left atrial mechanical stunning, after CV when sinus rhythm was restored, was evident by the decreased LAEF, which normalized within 10 days. Despite the significant LA functional recovery and LAEF normalization at 7-10 days after restoration of sinus rhythm, the LAEF was still significantly lower in patients with a history of paroxysmal or persistent AF compared with those with new-onset AF. A plausible explanation for the lower LAEF in patients with a prior AF history is a slower reverse remodeling which may be secondary to irreversible structural remodeling after their previous episodes of AF. These findings are consistent with another study reporting lower LAEF in patients with persistent or permanent $\mathrm{AF}$ as compared with paroxysmal $\mathrm{AF}$ and increased LAVI with the severity of AF type [21]. Moreover, LAEF seems to be significantly lower in persistent AF patients with arrhythmia recurrences after LA ablation versus those without, indicating that more extensive irreversible remodeling is an arrhythmia precursor [22].

In the present study, the LA peak longitudinal strain during reservoir and conduit phase prior to $\mathrm{CV}$ were lower 


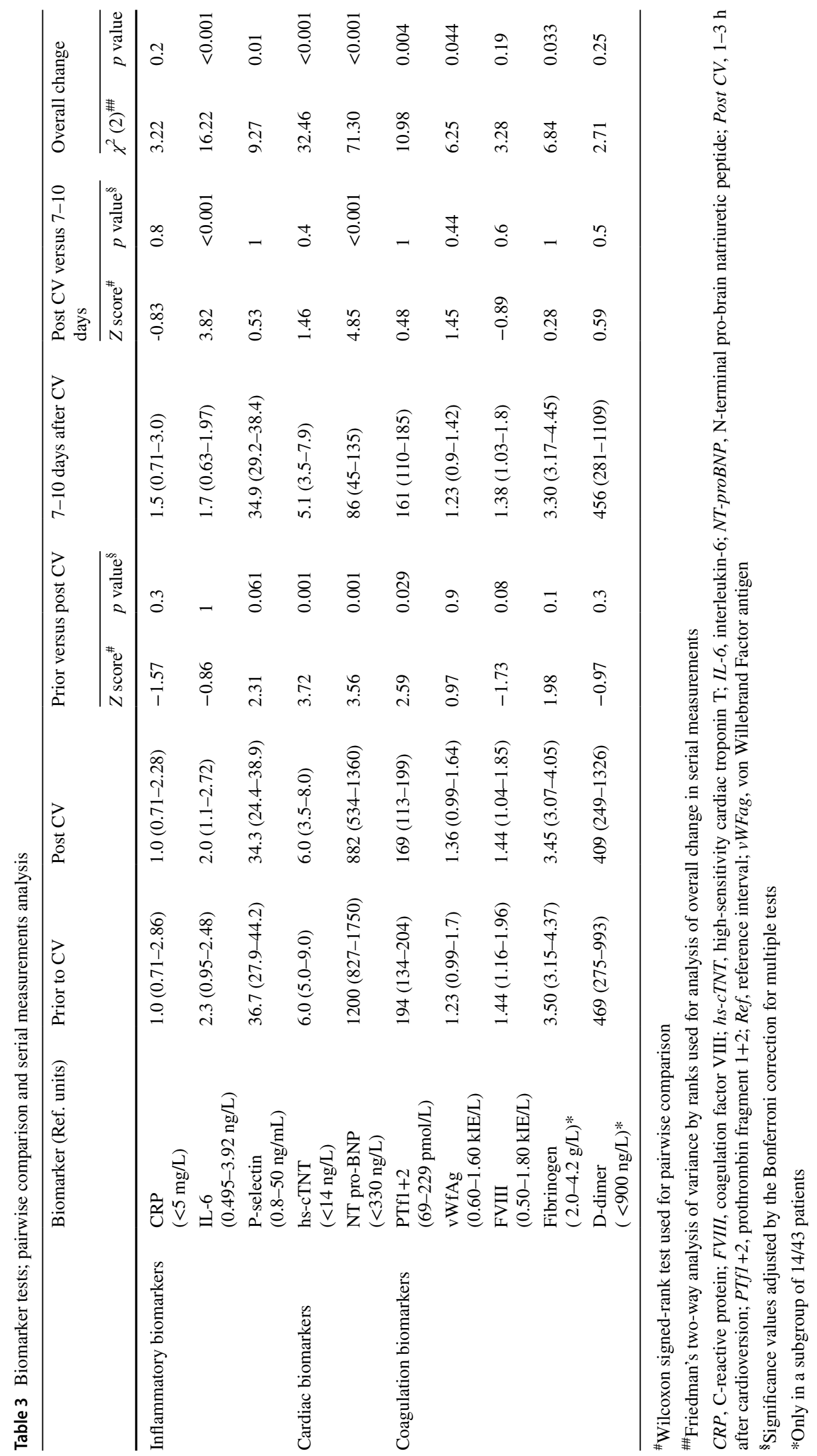


Fig. 3 Biomarkers association with white matter hyperintensities. Graphs represent ordinary mean plots and interaction plots, error bars, $95 \%$ confidence interval; CRP: C-reactive protein; hs-cTNT: high-sensitivity cardiac troponin-T; NTproBNP: N-terminal pro-brain natriuretic peptide; $\mathrm{PTf} 1+2$ : prothrombin fragment $1+2$; WMH: white matter hyperintensities.

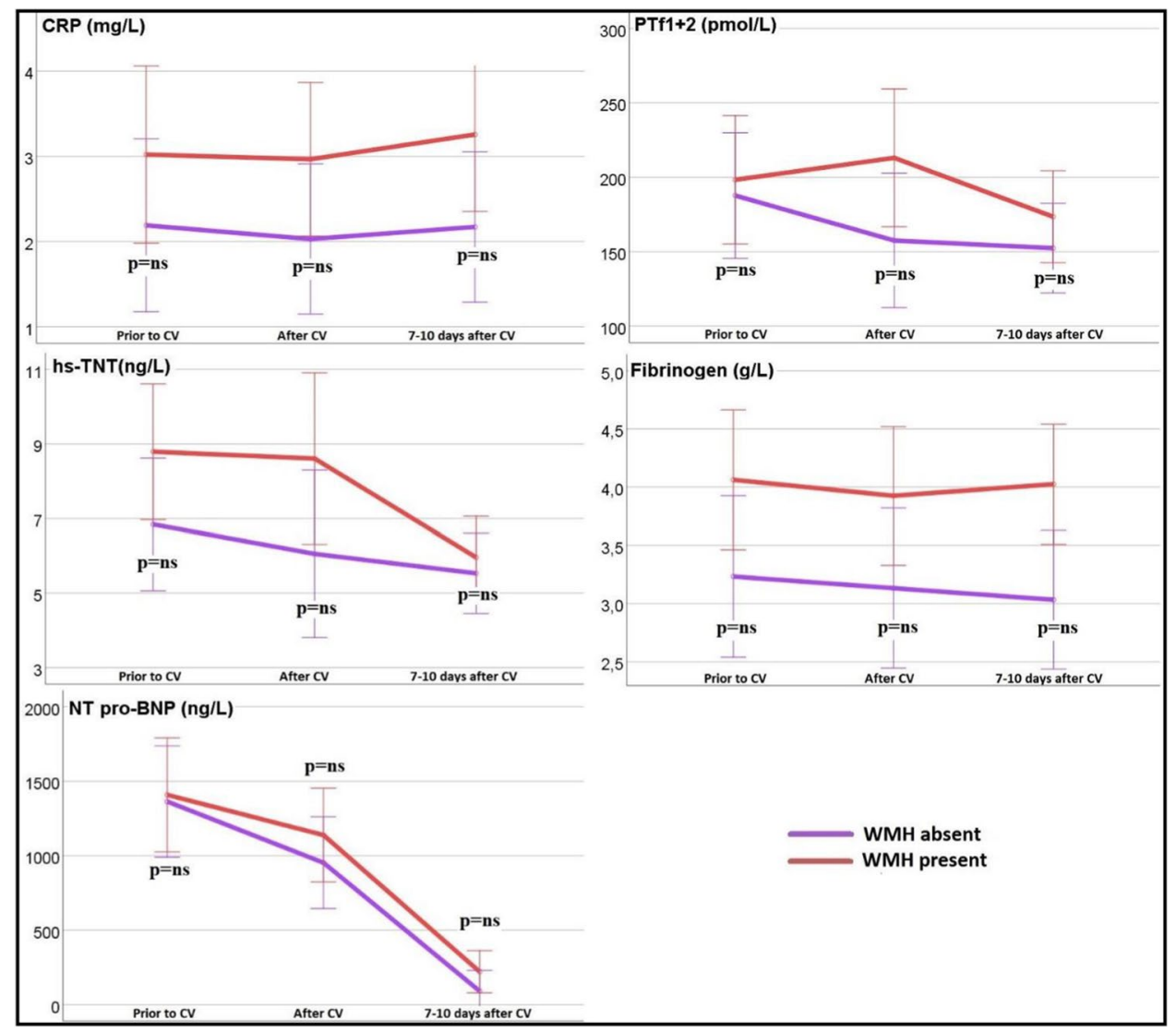

in patients with previous AF history as compared to those without. Although $\mathrm{LA} \varepsilon \mathrm{R}, \mathrm{LA} \varepsilon \mathrm{CD}$, and $\mathrm{LA} \varepsilon \mathrm{CT}$ improved significantly, they failed to normalize in both of these patient groups at 7-10 days. The presence of irreversible remodeling and fibrosis, identified during electro-anatomical mapping as low-voltage zones, have in other studies been associ-

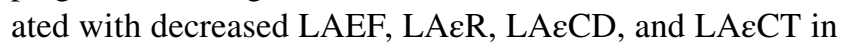
paroxysmal AF patients [23]. Moreover, extensive LA late gadolinium enhancement (LGE) on cardiac MRI, reflecting fibrosis, was inversely associated with LA emptying fraction, longitudinal strain during reservoir, conduit, and contractile phases; the latter assessed with cardiac MRI feature-tracking corresponding to STE analysis [24]. Left atrial deformation indices are strongly associated with LA fibrosis and AF progression, and as decreased indices are observed in patients with cryptogenic stroke, these early signs of LA remodeling may be a used to identify or predict a thrombogenic milieu [23-26].

Endothelial/tissue dysfunction and hypercoagulability, the remaining two elements of Virchow's triad, correspond to inflammatory/cardiac biomarkers and coagulation biomarkers respectively. The observation of the significant overall decrease of IL-6, P-selectin, hs-cTNT, NTproBNP, PTf1+2, and fibrinogen, and the significant transient increase of $\mathrm{vWfAg}$, from the time while in $\mathrm{AF}$ to the time of reverse remodeling after restoration of sinus rhythm is consistent with previous reports [2,27]. Elevated levels of IL-6 and CRP, indicating inflammatory activity, were not only associated with AF severity, but were also identified as prognostic markers for $\mathrm{AF}$ recurrence after $\mathrm{CV}$ and catheter ablation, as well as for stroke.[2, 27] Elevated CRP and IL-6 levels have previously been associated with increased LA size and long-lasting AF episodes indicating an association to LA structural changes and remodeling [28]. The maintenance of sinus rhythm after cardioversion resulted in a gradual decrease of CRP suggesting that inflammation is a consequence of AF [29].

Higher troponin levels and elevated NTpro-BNP have been associated with incident $\mathrm{AF}$, with $\mathrm{AF}$ recurrence after $\mathrm{CV}$, and with thromboembolic risk [30-34]. A sustained increase in troponin levels after CV has also been associated with AF recurrence, although the pathophysiologic mechanism is not clearly understood [30,35]. A hypercoagulability state in AF, as was reflected by elevated PTf1+2, fibrinogen, and vWfAg in the present study, has previously been reported even during the first $24 \mathrm{~h}$ of paroxysmal AF, observations that are in line with the current study [36-40].

Moreover, in the present study, a non-significant trend for higher CRP, hs-cTNT, NTpro-BNP, and PTf1+2 values was found in patients with brain WMH. Although CRP and 
Pf1+2 have been associated with the presence and severity of WMH in the elderly, and hs-cTNT and fibrinogen with the presence of WMH in patients with acute ischemic stroke, little is known about their relation to $\mathrm{WMH}$ in the AF population without stroke [41-47]. Since AF is associated not only to the presence of WMH, but also to the activation of inflammatory and coagulation systems, the findings in the present study further support the perception that $\mathrm{AF}$ is a marker of a cardiovascular disease, linking $\mathrm{AF}$ and $\mathrm{WMH}$, beyond thromboembolism [13, 14, 48, 49].

\subsection{Limitations}

The major limitation in the present study is the small size of the cohort and the underrepresentation of women. Moreover, left atrial volumes were measured only using the 4-chamber view and left ventricular volumes were measured as a mean of three consecutive beats during AF. Finally, the observed changes in inflammatory and coagulation biomarkers, although significant, were very small, underlining that the analysis of circulating biomarker should be interpreted with caution and used as hypothesis generating.

\section{Conclusion}

The observation of left atrial mechanical stunning followed by reverse functional remodeling in conjunction with the transiently higher level of inflammatory and coagulative biomarkers during AF might support the presence of an enhanced thrombogenicity even in patients with an inherent low risk for stroke. The finding of a more pronounced mechanical stunning in patients with a prior history of atrial fibrillation further supports the concept of adequate anticoagulation pericardioversion even in low-risk patients.

Author contribution Panagiotis Arvanitis: conceptualization, methodology, formal analysis, investigation, data curation, writing - original draft, writing-review and editing, visualization, project administration. Anna-Karin Johansson: investigation, data curation, writing - review and editing. Mats Frick: investigation, resources, writing-review and editing. Helena Malmborg: investigation, writing—review and editing. Spyridon Gerovasileiou: investigation: echocardiography, writing-review and editing. Elna-Marie Larsson: investigation: brain magnetic resonance imaging, writing —review and editing. Carina Blomström-Lundqvist: conceptualization, methodology, ethics application, formal analysis, investigation, resources, writing - review and editing, supervision, project administration, funding acquisition.
Supplementary Information The online version contains supplementary material available at https://doi.org/10.1007/s10840-021-01078-9.

Availability of data and material ClinicalTrials.gov Identifier: NCT02955004. Data available upon request.

Code availability Not applicable

Funding Open access funding provided by Uppsala University. The study was funded through unrestricted grants from the Swedish HeartLung Foundation, the Swedish Research Council, Correvio International Sárl (Geneva, Switzerland), and Selanders Stiftelse. No sponsor had any role in the design and conduct of the study; collection, management, analysis, and interpretation of the data; preparation, review, or approval of the manuscript; and decision to submit the manuscript for publication.

\section{Declarations}

Ethics approval This study was performed in line with the principles of the Declaration of Helsinki and was approved by the Swedish Ethical Review Authority.

Consent to participate Written informed consent was obtained from each study participant.

Consent for publication Written informed consent was obtained from each study participant.

Conflict of interest Authors Panagiotis Arvanitis, Anna-Karin Johansson, Mats Frick, Helena Malmborg, Spyridon Gerovasileiou, and ElnaMarie Larsson have no conflict of interest to declare that are relevant to the content of this article. Carina Blomström-Lundqvist has received research grants from Medtronic and speaker honorarium from Boston Scientific, Bayer, Sanofi, Pfizer, Johnson-Johnson, and Cathprint.

Open Access This article is licensed under a Creative Commons Attribution 4.0 International License, which permits use, sharing, adaptation, distribution and reproduction in any medium or format, as long as you give appropriate credit to the original author(s) and the source, provide a link to the Creative Commons licence, and indicate if changes were made. The images or other third party material in this article are included in the article's Creative Commons licence, unless indicated otherwise in a credit line to the material. If material is not included in the article's Creative Commons licence and your intended use is not permitted by statutory regulation or exceeds the permitted use, you will need to obtain permission directly from the copyright holder. To view a copy of this licence, visit http://creativecommons.org/licenses/by/4.0/.

\section{References}

1. Virchow R. Thrombosis and Emboli:(1846-1856). Canton: Science History Publ. 1998, vi p234.

2. Ding WY, Gupta D, Lip GYH. Atrial fibrillation and the prothrombotic state: revisiting Virchow's triad in 2020 (in eng). Heart. 2020;106(19):1463-8. https://doi.org/10.1136/heart jnl-2020-316977.

3. Staerk L, Sherer JA, Ko D, Benjamin EJ, Helm RH. Atrial fibrillation: epidemiology, pathophysiology, and clinical outcomes, (in eng). Circ Res. 2017;120(9):1501-17. https://doi.org/10.1161/ CIRCRESAHA.117.309732. 
4. Khan IA. Atrial stunning: basics and clinical considerations, (in eng). Int J Cardiol. 2003;92(2-3):113-28. https://doi.org/10.1016/ s0167-5273(03)00107-4.

5. Thomas L, Abhayaratna WP. Left atrial reverse remodeling: mechanisms, evaluation, and clinical significance, (in eng). JACC Cardiovasc Imaging. 2017;10(1):65-77. https://doi.org/10.1016/j. jemg.2016.11.003.

6. Thijssen VLJL, Ausma J, Borgers M. Structural remodelling during chronic atrial fibrillation: act of programmed cell survival. Cardiovascular Res. 2001;52(1):14-24. https://doi.org/10.1016/ s0008-6363(01)00367-4.

7. Iwasaki YK, Nishida K, Kato T, Nattel S. Atrial fibrillation pathophysiology: implications for management, (in eng). Circulation. 2011;124(20):2264-74. https://doi.org/10.1161/CIRCULATIO NAHA.111.019893.

8. Berger M, Schweitzer P. Timing of thromboembolic events after electrical cardioversion of atrial fibrillation or flutter: a retrospective analysis, (in eng). Am J Cardiol. 1998;82(12):1545-7, A8.

9. Nattel S, Opie LH. Controversies in atrial fibrillation, (in eng). Lancet. 2006;367(9506):262-72. https://doi.org/10.1016/S01406736(06)68037-9.

10. Airaksinen KE, et al. Thromboembolic complications after cardioversion of acute atrial fibrillation: the FinCV (Finnish CardioVersion) study, (in eng). J Am Coll Cardiol. 2013;62(13):1187-92. https://doi.org/10.1016/j.jacc.2013.04.089.

11. Watson T, Shantsila E, Lip GY. Mechanisms of thrombogenesis in atrial fibrillation: Virchow's triad revisited, (in eng). Lancet. 2009;373(9658):155-66. https://doi.org/10.1016/S0140-6736(09) 60040-4.

12. Vázquez M, et al. Assessment of silent microembolism by magnetic resonance imaging after cardioversion in atrial fibrillation, in englspa. Rev Esp Cardiol (Engl Ed). 2012;65(2):139-42. https:// doi.org/10.1016/j.recesp.2011.08.017.

13. Moroni F, Ammirati E, Rocca MA, Filippi M, Magnoni M, Camici PG. Cardiovascular disease and brain health: focus on white matter hyperintensities, (in eng). Int J Cardiol Heart Vasc. 2018;19:63-9. https://doi.org/10.1016/j.ijcha.2018.04.006.

14. Kobayashi A, Iguchi M, Shimizu S, Uchiyama S. Silent cerebral infarcts and cerebral white matter lesions in patients with nonvalvular atrial fibrillation, (in eng). J Stroke Cerebrovasc Dis. 2012;21(4):310-7. https://doi.org/10.1016/j.jstrokecerebrovasdis. 2010.09.004.

15. P. Arvanitis et al. Serial magnetic resonance imaging after electrical cardioversion of recent onset atrial fibrillation in anticoagulant-naïve patients - a prospective study exploring clinically silent cerebral lesions. J Atr Fibrillation. 2020;13 2.

16. Lang RM, et al. Recommendations for cardiac chamber quantification by echocardiography in adults: an update from the American Society of Echocardiography and the European Association of Cardiovascular Imaging, (in eng). Eur Heart J Cardiovasc Imaging. 2015;16(3):233-70. https://doi.org/10.1093/ehjci/jev014.

17. Badano LP, et al. Standardization of left atrial, right ventricular, and right atrial deformation imaging using two-dimensional speckle tracking echocardiography: a consensus document of the EACVI/ASE/Industry Task Force to standardize deformation imaging, (in eng). Eur Heart J Cardiovasc Imaging. 2018;19(6):591-600. https://doi.org/10.1093/ehjci/jey042.

18. Fazekas F, Chawluk JB, Alavi A, Hurtig HI, Zimmerman RA. MR signal abnormalities at $1.5 \mathrm{~T}$ in Alzheimer's dementia and normal aging, (in eng). AJR Am J Roentgenol. 1987;149(2):351-6. https://doi.org/10.2214/ajr.149.2.351.

19. Jakobsen JC, Gluud C, Wetterslev J, Winkel P. When and how should multiple imputation be used for handling missing data in randomised clinical trials - a practical guide with flowcharts.
BMC Med Res Methodol. 2017;17(1):162. https://doi.org/10. 1186/s12874-017-0442-1.

20. Lip GY, Nieuwlaat R, Pisters R, Lane DA, Crijns HJ. Refining clinical risk stratification for predicting stroke and thromboembolism in atrial fibrillation using a novel risk factor-based approach: the euro heart survey on atrial fibrillation, (in eng). Chest. 2010;137(2):263-72. https://doi.org/10.1378/chest.09-1584.

21. Lenart-Migdalska A, et al. Assessment of left atrial function in patients with paroxysmal, persistent, and permanent atrial fibrillation using two-dimensional strain, (in eng). J Atr Fibrillation. 2019;12(3):2148. https://doi.org/10.4022/jafib.2148.

22. Akkaya $\mathrm{E}$, et al. Clinical outcome and left atrial function after left atrial roof ablation using the cryoballoon technique in patients with symptomatic persistent atrial fibrillation, (in eng). Int J Cardiol. 2019;292:112-8. https://doi.org/10.1016/j.ijcard.2019.04. 091.

23. Chen Y, et al. Assessment of left atrial remodeling in paroxysmal atrial fibrillation with speckle tracking echocardiography: a study with an electrophysiological mapping system, (in eng). Int J Cardiovasc Imaging. 2019;35(3):451-9. https://doi.org/10.1007/ s10554-018-1470-6.

24. Habibi M, et al. Association of left atrial function and left atrial enhancement in patients with atrial fibrillation: cardiac magnetic resonance study, (in eng). Circ Cardiovasc Imaging. 2015;8(2):e002769. https://doi.org/10.1161/CIRCIMAGING. 114.002769 .

25. Yoon YE, et al. Echocardiographic predictors of progression to persistent or permanent atrial fibrillation in patients with paroxysmal atrial fibrillation (E6P Study), (in eng). J Am Soc Echocardiogr. 2015;28(6):709-17. https://doi.org/10.1016/j.echo.2015.01. 017.

26. Leong DP, et al. Left atrial dysfunction in the pathogenesis of cryptogenic stroke: novel insights from speckle-tracking echocardiography, (in eng). J Am Soc Echocardiogr. 2017;30(1):71-79. e1. https://doi.org/10.1016/j.echo.2016.09.013.

27. Guo Y, Lip GY, Apostolakis S. Inflammation in atrial fibrillation, (in eng). J Am Coll Cardiol. 2012;60(22):2263-70. https://doi.org/ 10.1016/j.jacc.2012.04.063.

28. Psychari SN, Apostolou TS, Sinos L, Hamodraka E, Liakos G, Kremastinos DT. Relation of elevated C-reactive protein and interleukin-6 levels to left atrial size and duration of episodes in patients with atrial fibrillation. Am J Cardiol. 2005;95(6):764-7.

29. Kallergis EM, et al. The role of the post-cardioversion time course of hs-CRP levels in clarifying the relationship between inflammation and persistence of atrial fibrillation. Heart. 2008;94(2):200-4.

30. Costabel JP, Burgos LM, Trivi M. The significance of troponin elevation in atrial fibrillation, (in eng). J Atr Fibrillation. 2017;9(6):1530.

31. Providência R, Paiva L, Barra S, Faustino A. Troponin rise in patients with atrial fibrillation: a marker of adverse prognosis and increased thromboembolic risk. Int J Cardiol. 2013;168(5):4889.

32. Lippi G, Picanza A, Formentini A, Bonfanti L, Cervellin G. The concentration of troponin I is increased in patients with acuteonset atrial fibrillation. Int J Cardiology. 2014;173(3):579-80.

33. Hussein AA, et al. Serial measures of cardiac troponin T levels by a highly sensitive assay and incident atrial fibrillation in a prospective cohort of ambulatory older adults. Heart rhythm. 2015;12(5):879-85.

34. Kim BS, et al. Usefulness of high-sensitivity troponin i to predict outcome in patients with newly detected atrial fibrillation, (in eng). Am J Cardiol. 2020;125(5):744-50. https://doi.org/10. 1016/j.amjcard.2019.12.004.

35. Hijazi Z, et al. High-sensitivity troponin I for risk assessment in patients with atrial fibrillation: insights from the Apixaban for Reduction in Stroke and other Thromboembolic 
Events in Atrial Fibrillation (ARISTOTLE) trial. Circulation. 2014;129(6):625-34.

36. Negreva M, Vitlianov K, Tasheva R. 073_16773-H1 early markers of coagulation activity prothrombin fragments $1+2$ and fibrinopeptide A are significantly increased in patients with paroxysmal atrial fibrillation. JACC: Clin Electrophysiol. 2017;3(10S):S23-3. https://doi.org/10.1016/j.jacep.2017.09.098.

37. Lip GY, Lowe GD, Rumley A, Dunn FG. Fibrinogen and fibrin D-dimer levels in paroxysmal atrial fibrillation: evidence for intermediate elevated levels of intravascular thrombogenesis. Am Heart J. 1996;131(4):724-30.

38. Sohara H, Amitani S, Kurose M, Miyahara K. Atrial fibrillation activates platelets and coagulation in a time-dependent manner: a study in patients with paroxysmal atrial fibrillation, (in eng). J Am Coll Cardiol. 1997;29(1):106-12. https://doi.org/10.1016/ s0735-1097(96)00427-5.

39. Li-Saw-Hee FL, Blann AD, Gurney D, Lip GY. Plasma von Willebrand factor, fibrinogen and soluble P-selectin levels in paroxysmal, persistent and permanent atrial fibrillation. Effects of cardioversion and return of left atrial function. Eur Heart J. 2001;22(18):1741-7.

40. Heeringa $\mathbf{J}$, et al. A longitudinal population-based study of prothrombotic factors in elderly subjects with atrial fibrillation: the Rotterdam Study 1990-1999, (in eng). J Thromb Haemost. 2006;4(9):1944-9. https://doi.org/10.1111/j.1538-7836.2006. 02115.x.

41. Wei C, Zhang S, Liu J, Yuan R, Liu M. Relationship of cardiac biomarkers with white matter hyperintensities in cardioembolic stroke due to atrial fibrillation and/or rheumatic heart disease, (in eng). Medicine (Baltimore). 2018;97(33):e11892. https://doi.org/ 10.1097/MD.0000000000011892.

42. von Rennenberg R, et al. High-sensitivity cardiac troponin $\mathrm{T}$ and severity of cerebral white matter lesions in patients with acute ischemic stroke, (in eng). J Neurol. 2019;266(1):37-45. https:// doi.org/10.1007/s00415-018-9085-3.
43. Dadu RT, et al. Cardiovascular biomarkers and subclinical brain disease in the atherosclerosis risk in communities study, (in eng). Stroke. 2013;44(7):1803-8. https://doi.org/10.1161/STROK EAHA.113.001128.

44. Satizabal CL, Zhu YC, Mazoyer B, Dufouil C, Tzourio C. Circulating IL-6 and CRP are associated with MRI findings in the elderly: the 3C-Dijon Study, (in eng). Neurology. 2012;78(10):720-7. https://doi.org/10.1212/WNL.0b013e3182 $48 \mathrm{e} 50 \mathrm{f}$.

45. Nagai K, Kozaki K, Sonohara K, Akishita M, Toba K. Relationship between interleukin- 6 and cerebral deep white matter and periventricular hyperintensity in elderly women, (in eng). Geriatr Gerontol Int. 2011;11(3):328-32. https://doi.org/10.1111/j.14470594.2010.00686.x.

46. Nagai M, Hoshide S, Kario K. Association of prothrombotic status with markers of cerebral small vessel disease in elderly hypertensive patients, (in eng). Am J Hypertens. 2012;25(10):1088-94. https://doi.org/10.1038/ajh.2012.85.

47. You CJ, Liu D, Liu LL, Liu QQ, Li GZ. Correlation between fibrinogen and white matter hyperintensities among nondiabetic individuals with noncardiogenic ischemic stroke, (in eng). J Stroke Cerebrovasc Dis. 2018;27(9):2360-6. https://doi.org/10. 1016/j.jstrokecerebrovasdis.2018.04.025.

48. Mayasi Y, et al. Atrial fibrillation is associated with anterior predominant white matter lesions in patients presenting with embolic stroke, (in eng). J Neurol Neurosurg Psychiatry. 2018;89(1):6-13. https://doi.org/10.1136/jnnp-2016-315457.

49. Gaita F, et al. Prevalence of silent cerebral ischemia in paroxysmal and persistent atrial fibrillation and correlation with cognitive function, (in eng). J Am Coll Cardiol. 2013;62(21):1990-7. https://doi.org/10.1016/j.jacc.2013.05.074.

Publisher's note Springer Nature remains neutral with regard to jurisdictional claims in published maps and institutional affiliations. 